\title{
PEEKING INTO THE BLACK BOX: SOME ART AND SCIENCE TO VISUALIZING AGENT-BASED MODELS
}

\author{
Stephen M. Guerin \\ RedfishGroup \\ 624 Agua Fria Street \\ Santa Fe, NM 87501, U.S.A.
}

\begin{abstract}
This paper explores current metaphors for visualizing agent-based models. Metaphors include grid, network, ndimensional cubes and landscape visualization techniques. A final section offers some theory underlying visualization of complex systems models with emphasis on mappings to non-equilibrium systems; conserved quantities and their flows; identifying order parameters and control parameters; and the presentation of phase transitions.
\end{abstract}

\section{INTRODUCTION}

Agent-based modeling (ABM) methodologies have matured over the last ten years. The increasing variety of ABM software toolsets (Gilbert \& Bankes 2002) and the accelerating availability of serious desktop computing power make modeling accessible to researchers with minimal investments in specialized programming skills and hardware expense. Most ABM implementations have at least a minimal delivery of visualization in addition to graphing and statistics collection. This paper will outline current visualization approaches and will give examples from the author's projects. The final section will explore potential theory that may inform future developments in visualization methodologies.

The ABM community is a subset of the greater simulation community. ABM models tend to use a relatively large number of thin agents which have simple internal rules and a high degree of interactions between agents (Parunak 1997). A second community within the simulation community is Multi-agent Systems (MAS). MAS, in contrast to ABM, tend to have fewer agents but use thicker agents which tend to have sophisticated internal machine learning rules. Over the last couple years, attendance at these two communities' respective conferences have become more intermixed. While the distinction is fading, the visualization techniques and theory outlined below will be specifically tuned to present ABM-type models where macro-level dynamics and structure are primarily caused by the interactions of a relatively high number of thin agents.

\section{GENRES IN THE ART OF VISUALIZING AGENT-BASED MODELS}

\subsection{Grid Visualization}

Perhaps the most familiar form of visualizing agent-based models is the grid method where agents occupy sites on a uniform 2D array of cells. The Sugarscape model (Epstein \& Axtell 1996) is a good example of this approach where lightweight agents interact in an active environment. Agent properties can be mapped to size and color in the visualization while properties specific to the environment or cell are represented with color. The grid approach can be extended to allow for 3D agents with additional model properties mapped to their motions. Figure 1 illustrates a grid visualization example from DrugSim (Agar et al. 2004). Here, cell color maps to the presence of drug supply and Agent color (blue) maps to a exceeding a risk threshold for using a drug.

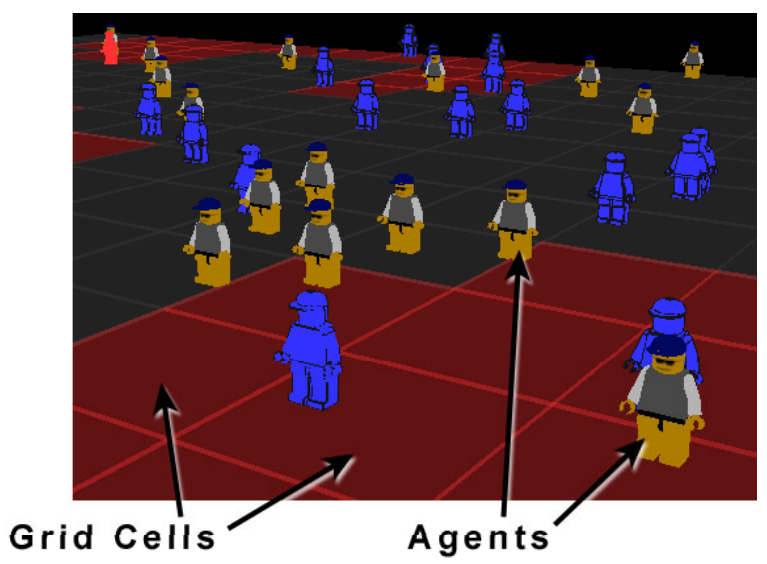

Figure 1: 3D Grid Visualization Example

A recent extension of the grid visualization approach is to move agents from interacting on a grid of abstract cells and toward agents interacting with data from geographic information systems (GIS). This is particularly useful as government agencies increasingly rely on GIS data formats as a 
lingua franca among agency computing systems. In the past, the scripting languages of the popular GIS environments were considered not expressive enough for anything but the simplest of ABM. As a result, model developers' only alternative was to use conventional ABM toolsets with featurelimited GIS plug-ins or libraries. However, recent releases of GIS toolsets now include extensions and internal programming support (Python, .NET, etc) that allow ABM models to be programmed directly in these environments. The next few years should see a broadening of ABM activity in this space as GIS practitioners seek to extend their toolsets with active processes like ABM on their mapsets. Figure 2 illustrates the combination of GIS with ABM. This model by Densmore, Guerin, Jung and McKenna explores effects of intervention during teenage cruising (black arrows as cars) around the Santa Fe plaza to mitigate disturbances to hotel guests (yellow blocks). Police intervention includes temporarily making streets one-way on cruising nights.

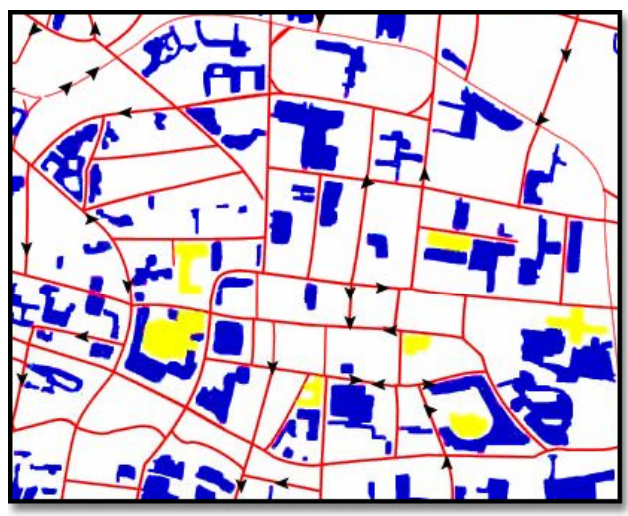

Figure 2: Extension of Grid Visualization to GIS

\subsection{Graph/Network Visualization}

Graphs are comprised of nodes and the edges or arcs that connect them. A grid is a kind of regular graph where each cell is a node with four or eight edges connecting it to its neighbors. Beginning in the late nineties, research into Small World (Watts \& Strogatz 1998) and Scale-free networks (Barabasi \& Albert 1999, Newman 2001) challenged conventional wisdom on the topologies of agent interactions being either regular or randomly distributed. An excellent review of research of Network Dynamics can be found in Newman (2003). The consequence of this research is that ABM modelers now include more sophisticated interaction graphs than grids and random graphs. With this new perspective, it is not uncommon for $\mathrm{ABM}$ models to have agents simultaneously interacting on multiple networks of varying topologies.

Graph layout, which is concerned with the many ways to arrange the nodes and edges for display, is an active area of research in graph theory (Junger, Mutzel, \& Junger 2003). A force-directed layout where nodes are connected by damped virtual springs, is one preferred method of layout for many of the author's visualizations. This method allows for the dynamic addition and removal of nodes. In addition, this layout method offers two additional animatable parameters to convey information: (1) $k$, the spring constant in Hooke's law:

$$
\mathrm{F}_{\mathrm{s}}=-k\left(x-x_{0}\right)
$$

and (2) $x_{0}$, the resting length of the spring. Figure 3 illustrates a force directed layout in three dimensions. The model, PeerPhoto, by Guerin and Kunkle, is a self-organizing peer-topeer data storage network where both storage nodes and data files are given agency. The nodes represent servers. Their size represents capacity and their color represents utilization. Edges between nodes represents the logical network by which data migrates. The latency between two nodes is mapped to the resting length of the spring that is used to layout an edge. The model also includes users (not shown) in a social network requesting data from the network and recommending data files to their neighbors.

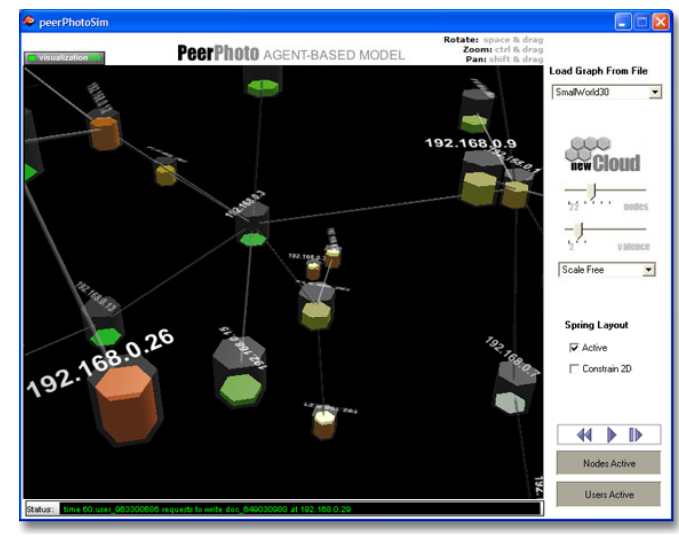

Figure 3 : 3D Graph Visualization

ABM visualization has benefited significantly from the popularity of computer gaming. To maximize gaming experience, most modern computers are now shipping with hardware graphics cards that dramatically accelerate 3D display. Additionally, deployment of 3D visualizations are now more reliable as most machines come with the rendering libraries OpenGL and/or DirectX pre-installed. One capability of both of these libraries is particle streams. Particle streams are used in games and movies for effects like fire, smoke, explosions, sparks and splashes. ABM visualizations can map to various parameters that govern particle stream dynamics including particle size, emitter frequency, particle color, particle lifetime, environmental wind, and environmental gravity. Figure 4 illustrates the use of agent particle streams in a visualization by Guerin and Kunkle. The model is of the Criminal Justice System of England and Wales which was modeled for the UK Parliament and Home Office for use in policy appraisal (Boyle et al. 2003). 


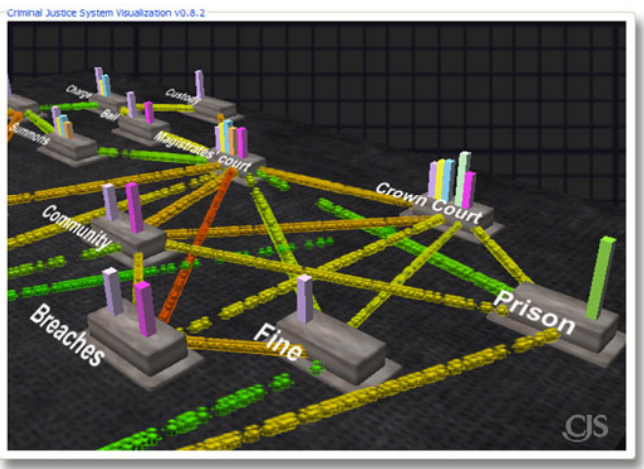

Figure 4: Parameterized Particle Streams on Graph Arcs

\subsection{Landscape Visualization}

Sewell Wright (1889-1988) conceived of landscapes as an appropriate metaphor for displaying the fitness of organisms during biological evolution. Kauffman (1993) extended the use of fitness landscapes in his work with NK networks. Landscapes are useful in ABM visualizations for the display of the energy landscapes (see section 3.2) or for displaying the performance of a many parameter settings when performing optimization. Landscapes are extensions to grid visualizations where cells are raised to an elevation according to a performance metric. Additionally, landscapes, as illustrated in Figure 5, can be used to map GIS information to the environment of a model. This wildfire simulation by Guerin and Wimberly uses web-based GIS to map elevation information to the landscape. Additional GIS layers map to the climate and fuel conditions of each cell in the landscape.

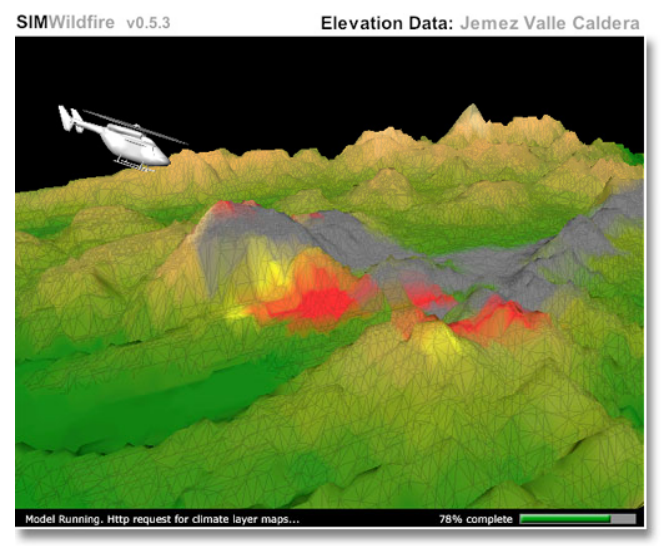

Figure 5: Landscape Visualization

\subsection{N-Dimensional Cube Visualization}

Both the parameter space and the performance space of ABM models tend to be high-dimensional. N-Dimensional cubes are useful for interactive exploration of these spaces. The cubes allow for dynamic mapping of parameters to cube axes and agent (or datapoint) sizes and colors. The user is able to dynamically change axes mappings and datapoint properties in order to visually data-mine for hidden relationships in the data.

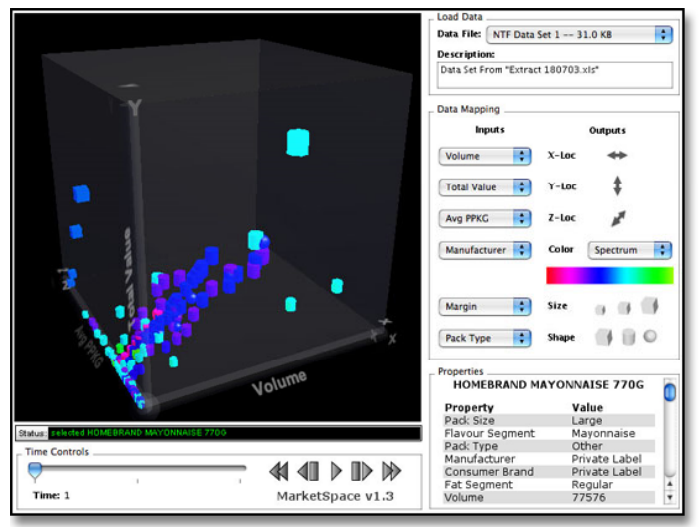

Figure 6: N-Dimensional Data Cube Example

All the metaphors and examples above illustrate some approaches to visualizing ABM. They are by no means exhaustive - Many techniques remain to be invented.

\section{SOME SCIENCE BEHIND THE VISUALIZATION OF AGENT-BASED MODELS AND COMPLEX ADAPTIVE SYSTEMS}

$\mathrm{ABM}$ is a toolset that has grown up around the research of complex adaptive systems (CAS) at the Santa Fe Institute and many other institutions around the world. Researchers employing ABM are usually interested in reproducing observed macroscopic dynamics by specifying microscopic rules and interactions (Epstein \& Axtell 1996, Bonabeau 2002). As of yet, there remains no single accepted definition or measure of Complexity. Many doubt there ever will be one.

Nonetheless, there are some general CAS themes from non-equilibrium thermodynamics and statistical mechanics that are worth expounding for the purpose of developing future visualization techniques. Both disciplines deal with systems of extreme numbers of interacting components and seek to describe statistical relationships between the aggregate macroscopic variables of these systems. Thermodynamics finds these relationships through empirical manipulation and observation while Statistical Mechanics derives the relationships from the interaction rules of the microscopic components. The relevance to ABM should be apparent as the field is also dealing with systems of many interacting components.

\subsection{Order/Control Parameters and Phase Transitions}

It is common for even simple ABM models to have tens of input parameters. Complicated models can often have a few hundred or even thousands. Similarly, the output per- 
formance measures can also number in the tens or hundreds for statistics that can be collected from a model run. A major question becomes "Which parameters should I let the user interactively adjust during a model run and which performance metrics should I visualize"?

Modelers of complex systems are typically interested in exploring nonlinear effects of input parameters and the performance of the system near points of great change (phase transitions). Visualizations can borrow from statistical physics in their use of order parameters and control parameters.

An order parameter summarizes the macroscopic state of the system. Order parameters are not typically directly manipulateable by the experimenter. Order parameters are ideal candidates for visualization as they effectively collapse the dimensionality of the system to perceptionally manageable numbers. Nonlinear changes in order parameters either during model runs or during parameter sweeps are indicative of phase transition. An example of an order parameter is the degree of magnetization in an Ising model.

Control parameters, on the other hand, are usually those parameters that are manipulateable by the experimenter and can be shown to move the order parameter through phase transitions. These parameters are good candidates for sliders or other GUI elements for direct user manipulation. An example of a control parameter is temperature in the Ising model. Finding the appropriate control parameter can be difficult. One approach is to first find some candidate order parameters and then parameter sweep input parameters and graph changes to the order parameters. However, with even ten input parameters, this poses a computational challenge. One approach to cut down on the search time is Design of Experiments (DOE) which is a technique that systematically varies the extreme ranges of parameters while monitoring changes to potential order parameters. Figure 7 is an example

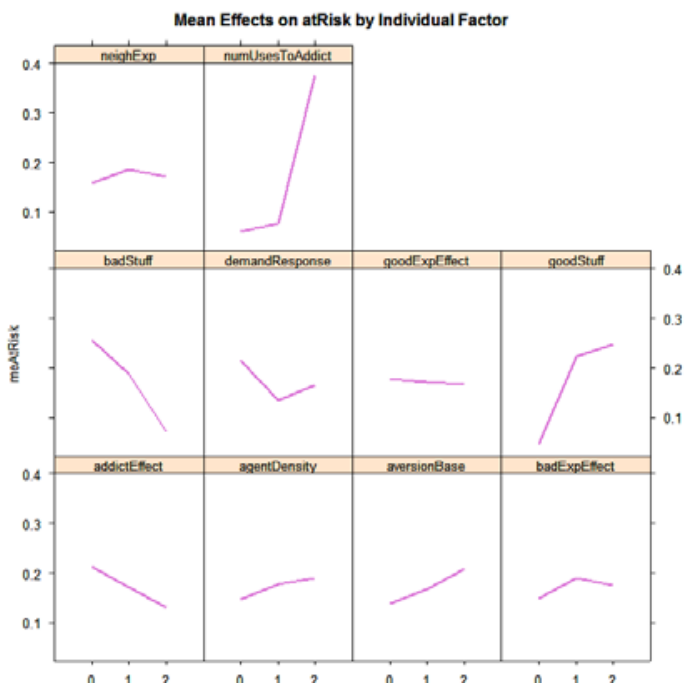

Figure 7: Use of Design of Experiments to Suggest Candidate Control Parameters from DrugSim (Agar et al. 2004). The matrix shows the effects of 10 parameters on the behavior of the order parameter (atRisk) in the model. Candidate control parameters will have steep positive or negative slopes. In this example, candidate control parameters are $n u$ mUsesToAddict, badStuff, and goodStuff.

\subsection{Non-Equilibrium Open Systems, Conserved Quantities and Flow}

An underlying theme in CAS research is that of structure creation. This section proposes that there may be universal principles for structure creation in ABM and what the consequences might be for visualization. These principles would not be dependent on particular details of a given model but would generalize models into a relatively few number of universality classes that share common microscopic descriptions that yield equivalent macroscopic dynamics.

Isolated physical systems tend toward equilibrium states in accordance with the second law of thermodynamics. Equilibrium is characterized by homogeneous distributions of conserved quantities which lack gradients from which work can be extracted (Kugler \& Turvey 1987). A consequence of the push toward equilibrium is that structures in the physical world, without maintenance, tend to decay over time.

If structure tends to spontaneously decay, how does it form in the first place? The theme of structure creation in CAS research is the idea that structure self-organizes as a system is driven far-from-equilibrium. In physics, a system is out of equilibrium if a concentration gradient exists for a conserved quantity (energy, charge, mass, linear momentum, angular momentum, etc.). Concentration gradients are the source of spontaneous flow processes in physics. These flows do physical work to move systems back to equilibrium. If a system is far enough from equilibrium, the system can spontaneously break symmetry and organize to dissipate the existing gradients. Prigogine (1962) referred to these as dissipative structures. Schneider and Kay (1995) offer a causal explanation that self-organizing structures come into existence because they are more efficient at dissipating gradients than are unorganized flows.

Previous work by the author has shown early evidence in an ant-foraging model that the second law and mechanisms of structure creation may apply to ABM in much the same way that they do in familiar real-world physical systems (Gambhir et al. 2004). As a simple example of a nonequilibrium flow process in ABM, consider a population of randomly walking agents in a space. If one initializes the model out of equilibrium, with the agents concentrated in one location, the agents will spontaneously diffuse to equilibrium concentrations where they become distributed throughout the space.

Redistribution of agent concentrations is not the only spontaneous flow toward equilibrium that can occur in ABM. Most models have some property or quantity trans- 
acted (1) among the agents; (2) among the agents and their environment; or (3) within the environment itself. For example, in social models, a quantity transacted may be an attitude or belief that spreads among the agents. In economic models the transacted quantity may be a currency or risk, or in ant foraging models it may be the pheromone in the environment.

We can use the Ising model as an example for defining conserved quantities in ABM. The Ising model is a simplified model for describing ferromagnetism and liquid-gas transitions. It is defined as a grid of "agents" with spins (orientations) that can be either up or down. Each time step, agents orient their spin to be in alignment with the majority of their neighbors. The conserved quantity, energy, is defined for each neighbor interaction. If the spins of two neighbors are the same, the local energy on that edge is -1 . If the spins of the agents are different, the energy is +1 . The summation of local energies across all the edges defines a Hamiltonian and is what is minimized during a model run. The state space of the model can be visualized as an energy landscape.

When visualizing a model, a first step might be to identify what the conserved quantities may be in the model and what might be the mechanism(s) for their transfer. The author has observed that in a general class of models that exhibit self-organization, there are at least two conserved quantities interacting during pattern formation. Additional work remains to make this claim more rigorous. But for now, a visualization artist might be interested to illustrate how these conserved quantities may couple or transact as the two are driven toward equilibrium. Some guidance can be taken from Onsager relations in thermodynamics that describe empirically derived coupling relations between the flow rates of two conserved quantities. These empirical relations are linear near equilibrium and go nonlinear as a system is driven further from equilibrium.

A useful way to think about structure formation in $\mathrm{ABM}$ is to consider all the locations in the model description and initial conditions that are not symmetric. Initial placement of agents, the initial allocation of resources to agents or interaction rules may be locations for initial or maintained asymmetries. The claim here is that these asymmetries drive organization in models and ABM visualizations can be used to illustrate the relationship of asymmetries to order creation.

A third potential for visualization could be tracking the degrees of freedom in the behavioral repertoire of agents. As a system self-organizes, components of the system are expected to lose degrees of freedom through the emergence of context-sensitive constraints (Guerin \& Kunkle, 2004; Juarrero, 1999; Kugler \& Turvey, 1987). Visualizing the increasing constraint on agents and their loss of degrees of freedom can be a methodology for visualization that illustrates mechanisms of self-organization at work.

\section{ACKNOWLEDGMENTS}

Ideas in this paper have formed from conversations with Michael Agar, Roger Critchlow, Owen Densmore, Manoj Gambhir, Richard Harris, Robert Holmes, Stuart Kauffman, Daniel Kunkle, Carl Tollander, Frank Wimberly, and members of the FRIAM Applied Complexity Group in Santa Fe, New Mexico <www. friam.org> .

\section{REFERENCES}

Agar, M., Guerin, S., Holmes, R., and Kunkle, D. 2004. DrugSim: the structure of agent-based revolutions. In: Submitted for Publication.

Barabási, A. L. and Albert, R. 1999. Emergence of scaling in random networks, Science 286, 509-512.

Bonabeau, E. 2002. Agent-based modeling: Methods and techniques for simulating human systems. Proceedings of the National Academy of Sciences of the United States of America 99 Suppl 3, 7280-7287.

Boyle, S., Guerin, S., Pratt, J., and Kunkle, D. 2003. Application of agent-based simulation to policy appraisal in the criminal justice system in England and Wales. In: Proceedings of Agent 2003:Challenges in Social Simulation. Chicago, IL.

Epstein, J. M. and Axtell, R. L. 1996. Growing Artificial Societies: Social Science from the Bottom Up. Cambridge, MA: MIT Press.

Gambhir, M., Guerin, S., Kauffman, S.,and Kunkle, D. 2004. Steps toward a possible theory of organization. In: Proceedings of International Conference on Complex Systems 2004. Boston, MA.

Gilbert, N. and Bankes, S. 2002 Platforms and methods for agent-based modeling. Proceedings of the National Academy of Sciences of the United States of America, 99 Suppl 3, 7197-7198.

Guerin, S. and Kunkle, D. 2004. Emergence of constraint in self-organizing systems. Journal of Nonlinear Dynamics, Psychology, and Life Sciences, 8 (2).

Juarrero, A. 1999. Dynamics in action: Intentional behavior as a complex System. Cambridge, MA: MIT Press.

Junger, M., Mutzel, P., and Junger, M. 2003. Graph Drawing Software (Mathematics and Visualization). New York: Springer-Verlag

Kauffman, S., 1993., The Origins of Order. Oxford: Oxford University Press.

Kugler, P. N. and Turvey, M. T. 1987. Information, Natural Law, and the Self-assembly of Rhythmic Movement. Hillsdale, NJ: Lawrence Erlbaum.

Newman, M. E. J. 2001., Clustering and preferential attachment in growing networks, Physical Review E 64, 025102.

Newman, M. E. J. 2003. The structure and function of complex networks, SIAM Review 45, 167-256. 
Parunak, H. V. D. 1997. Go to the ant: Engineering principles from natural multi-agent systems. Annals of $O p$ erations Research, 75, 69-101.

Prigogine, I. 1962. Non-equilibrium statistical Mechanics. New York: Wiley Interscience.

Schneider, E. D. and Kay, J. J. 1995. Order from disorder: the thermodynamics of complexity in biology. What is life? The next fifty years: Speculations on the future of biology In M. P. Murphy \& L. A. J. O'Neill (Eds.). 161-172. Cambridge: Cambridge University Press.

Watts, D. J. and Strogatz, S. H. 1998. Collective dynamics of 'small-world' networks. Nature 393,440-42.

\section{AUTHOR BIOGRAPHY}

STEPHEN M. GUERIN is currently president of RedfishGroup <www.redfish.com>. His work centers on the design and visualization of self-organizing systems. He recently worked as a Senior Software Developer at BiosGroup and participated as a member of Stuart Kauffman's research group. In the last 15 years he has delivered over 20 simulation-based applications for Fortune 100 and Government clients. RedfishGroup is based in Santa Fe, New Mexico. Stephen's email address is <stephen.guerin@redfish.com>. 